\title{
Detection of second-order nonlinear optical magnetization by mapping normalized Stokes parameters
}

\author{
Fabio Antonio Bovino, ${ }^{1}$ Maria Cristina Larciprete, ${ }^{2}$ Concita Sibilia, ${ }^{2, *}$ Gregory Leahu, ${ }^{2}$ \\ György Váró, ${ }^{3}$ and Csilla Gergely ${ }^{4,5}$ \\ ${ }^{1}$ Quantum Optics Lab Selex ES 16154, Via Puccini 2, Genova 16154, Italy \\ ${ }^{2}$ Dipartimento SBAI, Università di Roma La Sapienza, Via Scarpa 16, Roma 00161, Italy \\ ${ }^{3}$ Institute of Biophysics, Biological Research Center of the Hungarian Academy of Sciences, Szeged H-6701, Hungary \\ ${ }^{4}$ Université Montpellier 2, Laboratoire Charles Coulomb UMR 5221, Montpellier F-34095, France \\ ${ }^{5}$ CNRS, Laboratoire Charles Coulomb UMR 5221, Montpellier F-34095, France \\ ${ }^{*}$ Corresponding author: concita.sibilia@uniroma1.it
}

Received October 3, 2012; revised December 20, 2012; accepted January 9, 2013; posted January 14, 2013 (Doc. ID 177349); published February 14, 2013

\begin{abstract}
A measurable magnetic (nonlocal) contribution to the second harmonic generation (SHG) of nonmagnetic materials is an intriguing issue related to chiral materials, such as biomolecules. Here we report the detection of an intensity-dependent optically induced magnetization of a chiral bacteriorhodopsin film under femtosecond pulse excitation $(830 \mathrm{~nm})$ and far from the material's resonance. The analysis of the pump intensity-dependent noncollinear SHG signal, by means of the polarization map of normalized Stokes parameters, allows one to improve the detection of the nonlinear optical magnetization $M(2 \omega)$ contribution to the SHG signal. (c) 2013 Optical Society of America
\end{abstract}

OCIS codes: $\quad 190.4400,160.4330,160.1585$

\section{INTRODUCTION}

Chirality is equivalent to the absence of mirror plane symmetry and with this property the medium exhibits a natural optical activity, i.e., it rotates the plane of polarization of light [1-3]. The optical rotation occurs when the refractive indices of right and left circularly polarized light are unequal. A difference in the corresponding absorption indices causes planepolarized light to become elliptically polarized (circular dichroism). This property has acquired great importance in spectroscopy, biology, and crystallography, to name a few. To describe the linear optical response of an optically active medium, it becomes necessary to go beyond the electric dipole approximation and to include the induced magnetic dipole moment. A simplified model to explain the role of magnetic effects in the optical activity is founded on the electron path in a chiral molecule [4]: As the electrons of chiral molecules are displaced from their equilibrium by the application of the electromagnetic field, they are forced to move along a helicallike path. This process gives rise to an induced magnetic dipole moment of the molecules in addition to the electric dipole moment; therefore chiral molecules respond to both the electric and magnetic component of the field [1,2]. At a more microscopic level, for bio-molecules [3] , two principal mechanisms are responsible for a chiral molecule's optical activity: (i) one electron mechanism, in which the electric and the magnetic transitions are mixed on the same chromophore, due to its asymmetric environment and (ii) a coupled oscillators mechanism, in which two electric-dipole allowed transitions, belonging to two separate chromophores in the molecule, are asymmetrically coupled.
Although the optical activity exists in natural media, it can be an order of magnitude stronger in artificial structured media (chiral metamaterials) [믁], i.e., artificial materials designed to deliver an unusual electromagnetic response [8]. The optical activity effect is a linear effect, i.e., the angle of rotation of a polarized light beam or its ellipticity do not change with the intensity of the light. However, chiral materials can also exhibit nonlinear optical activity raised by the quadratic, cubic, and quartic functions of the electromagnetic field strength $[2,3,9]$.

Particularly interesting is the second harmonic (SH) optical activity, observed by means of different techniques [2]. For instance, the optical rotatory dispersion, i.e., the rotation angle of the second harmonic generation (SHG) field in comparison with an achiral material, is defined as $\vartheta_{\mathrm{ORD}}=\tan ^{-1}\left(E_{s}(2 \omega) / E_{p}(2 \omega)\right)$ and obtained by using linearly polarized fundamental fields. Another technique is the detection of SHG circular dichroism [2], i.e., the different nonlinear response from a pump beam with left circular (LC) and right circular (RC) polarization. Additionally, we would like to mention the detection of the SHG linear difference, for which there is no analogue in linear optics, determined by the diverse nonlinear $\mathrm{SH}$ response arising from a pump beam that is linearly polarized at $+45^{\circ}$ and $-45^{\circ}$, respectively. The above mentioned techniques represent an efficient tool to study the surface chirality, although with some limitations to discriminate the magnetic dipole or electric dipole interaction. Other methods, such as the continuous polarization method [2], prove very sensitive to detecting the nonlinear optical activity of materials in the form of films several wavelengths thick; in practice, it consists of the detection of the 
linear polarization state of the SHG as a function of the polarization state of the pump beam.

By using a method based on the linear polarization change of the pump beams under noncollinear configuration, we have analyzed the nonlinear optical activity of a film of chiral material: bacteriorhodopsin (BR).

The manuscript is organized as follows. We first briefly describe the BR sample. Then we present and discuss the utilized method and the experimental results. Finally, we give the conclusions.

\section{SHG-NONLINEAR OPTICAL ACTIVITY}

To discuss the nonlinear response of chiral materials, Kauranen et al. [10] introduced "nonlocal" or "multipolar" effects in the second-order nonlinear formalism. Several experimental results [11-13], indicate that magnetic contributions are necessary to accurately explain the nonlinear optical activity [2]. The second-order nonlinear polarization (at $2 \omega$ frequency) consists of a series of multipole terms [ㅁ, $\underline{10}, \underline{14}]$ :

$$
\vec{P}_{\mathrm{eff}}(2 \omega)=\vec{P}_{D}(2 \omega)-i \vec{k} \vec{Q}(2 \omega)-\frac{1}{\omega} \vec{k} \times \vec{M}(2 \omega)
$$

where $\vec{P}_{D}(2 \omega), \vec{Q}(2 \omega), \vec{M}(2 \omega)$ represent the electric and magnetic-dipole polarization, electric-quadrupole polarization, and nonlinear magnetization, respectively. The electric and magnetic-dipole polarization is given by $P_{i^{D}}(2 \omega)=$ $\sum_{j, k} \chi_{i j k}^{(2) e e e} E_{j}(\omega) E_{k}(\omega)+\chi_{i j k}^{(2) e e m} E_{j}(\omega) B_{k}(\omega)$, where $\chi_{i j k}^{(2) e e e}$ is the nonlinear electrical susceptibility tensor and $\chi_{i j k}^{(2) e e m}$ is the magnetic-dipole tensor. The quadrupole interaction, $\vec{Q}(2 \omega)$, is somewhat negligible; in any case it should be possible to incorporate quadrupole effects into the magnetic dipole terms, as they both have the same symmetry [1,2]. Finally, the nonlinear magnetization is $M_{i}(2 \omega)=$ $\sum_{j, k} \chi_{i j k}^{(2) m e e} E_{j}(\omega) E_{k}(\omega), \chi_{i j k}^{(2) m e e}$ being the magnetic susceptibility tensor. The nonlocal $\chi_{i j k}^{(2) m e e}$ and $\chi_{i j k}^{(2) e e m}$ susceptibilities are third-rank tensors; their symmetry differs from the local electric-dipolar susceptibility. According to [1], the superscripts of $\chi_{i j k}^{(2) e e e}, \chi_{i j k}^{(2) e e m}$, and $\chi_{i j k}^{(2) m e e}$ account for the two possible interactions, i.e., the electric dipole interaction $(e)$, or the magnetic interaction $(m)$; the last two superscripts indicate the interaction annihilating two input photons and the first indicates the interaction creating the output photon at the SH.

Within this context, we recently investigated the nonlinear optical activity of an oriented BR protein film $[\underline{13,15]}$, by means of a noncollinear SHG interaction. We chose BR since it has a well-established structure [15] and its crystal structure is known with a high degree of accuracy. Furthermore, the orientational averages connecting the retinal chromophore to the protein cage have been reported as the main responsible for SHG optical rotatory dispersion in BR [16]. To study $\mathrm{BR}$, we applied a method recently developed based on the simultaneous variation of the linear polarization state of both fundamental beams, while the incidence angle is fixed. Thus the noncollinear SH linearly polarized signal is represented as a function of the polarization states of both pump beams. The resulting polarization map displays a pattern that is characteristic of the investigated crystalline structure and offers the possibility to address several properties as the nonzero terms of the nonlinear optical tensor [17]: the ratio between the different nonzero elements of the nonlinear optical tensor [18] and the orientation of the optical axis [19], to name two. By applying this method, we estimated the value of all nonlinear elements of the susceptivity tensors of BR, including the one responsible for the second-order nonlinear magnetization. However, the nonlinear elements are given only as relative value: The method, in the present configuration, does not allow evaluating their absolute value.

In order to better analyze the influence of the nonlinear magnetization on the SHG signal and to evidence its detectability, we extended the above mentioned method to the detection of normalized Stokes parameters of the SH signal generated by the noncollinear interaction from the oriented BR film. A two-channel Stokes polarimeter setup has been realized. Aging at a fixed incidence angle, the polarization state of both fundamental beams is systematically varied and the normalized Stokes parameters of the generated signal are reported as a function of polarization states of both pump beams. The result is a polarization map of the normalized Stokes parameters at the $2 \omega$ frequency. The change of the linear input polarization state of the two pumps provides all information about the polarization content of the SH signal. The change of the pump intensity gives an additional parameter to improve the detection of the nonlinear optical magnetization, as much as to better understand its role in such kinds of chiral materials.

The method and the experimental results are discussed in the Section 4 .

\section{BACTERIORHODOPSIN FILM}

As also reported in the [13], the transmembrane protein BR is one of the simplest known active membrane transport system [20-24] with a well-established structure. Trimers of BR proteins are placed in a hexagonal two-dimension lattice within the purple membrane, thereby forming a real crystalline structure similar to a photonic crystal or to a "natural optical chiral metamaterial," belonging to the point group symmetry P3. Each BR monomer contains a covalently bound retinal chromophore, presenting its own transition dipole, which is responsible for its outstanding quadratic nonlinear optical response enhanced by the protein environment [22], as well as for the light absorption [15] in the visible range. The chromophore retinal axis is oriented at an angle of $23^{\circ} \pm 4^{\circ}$ with respect to the plane of the purple membrane so to form an isotropic conical polar distribution around the normal [15].

The analyzed oriented BR film was prepared by using the asymmetric electrostatic interaction of the surface charge of the membrane fragments with a charged support surface. The electrophoretic deposition technique allows growing a $4 \mu \mathrm{m}$ thick oriented film onto a substrate covered by a $60 \mathrm{~nm}$ thick ITO film. The resulting BR films, composed by $~ 800$ purple membrane layers (of $5 \mathrm{~nm}$ thickness each), were characterized in terms of homogeneity, optical, and electrical properties.

The optical properties of BR film have been detected by a spectrophotometer. Dispersion of the refractive index of $\mathrm{BR}$, $n(\lambda)$, was taken from [25], i.e., $n_{\omega}=1.526, n_{2 \omega}=1.549$, while the extinction coefficients of $k_{2 \omega}=0.002$ and $k_{\omega}=5 \cdot 10^{-4}$ were obtained from the linear spectra of BR film. The spectrophotometric analysis also indicates a negligible birefringence. 
Table 1. Nonlinear Coefficients Retrieved at the Input Pump Power of $125 \mathrm{~mW}^{a}$

\begin{tabular}{lccc}
\hline \multicolumn{4}{c}{ Nonlinear Coefficients at $125 \mathrm{~mW}$} \\
\hline & $\chi_{i j k}^{(2) e e e}$ & $\chi_{i j k}^{(2) e e m}$ & $\chi_{i j k}^{(2) m e e}$ \\
\hline$\chi_{x y z}=\chi_{x z y}$ & $-0.1-0.08 i$ & $0.0009+4.5 .10^{-3} i$ & $0.03+0.06 i$ \\
$\chi_{x z x}=\chi_{x z x}$ & $0.45+0.008 i$ & $0.0002+3.6 .10^{-2} i$ & $0.06+0.05 i$ \\
$\chi_{y y z}=\chi_{y z y}$ & $0.45+0.008 i$ & $0.0045+5.4 .10^{-2} i$ & $0.06+0.05 i$ \\
$\chi_{y x z}=\chi_{y z x}$ & $0.1+0.08 i$ & $-0.0009-4.5 .10^{-3} i$ & $-0.03-0.06 i$ \\
$\chi_{z x x}$ & 0.5 & $4.5 .10^{-3} i$ & $0.0009 i$ \\
$\chi_{z y y}$ & 0.5 & $4.5 .10^{-3} i$ & $0.026 i$ \\
$\chi_{z z z}$ & 1 & $4.5 .10^{-3} i$ & $0.045 i$ \\
$\chi_{z x y}=\chi_{z y x}$ & 0 & $2.27 .10^{-2} i$ & 0 \\
\hline
\end{tabular}

${ }^{a}$ The values are normalized to $\chi^{e e e} z z z$.

$\mathrm{BR}$ reveals a chiral behavior with a $\mathrm{SH}$ signal that contains a contribution from the second-order nonlinear magnetization. The elements of the nonlinear coefficients, including the one responsible for the nonlinear magnetic polarization, are here reported in the Table $\underline{1}$.

\section{NONCOLLINEAR SHG AND STOKES PARAMETERS}

In order to study the full polarization behavior of the SHG signal, a polarization resolved technique based on the detection of Stokes parameters becomes necessary. Polarization resolved SHG (see [26] and references therein) such as nonlinear optical Stokes ellipsometry [27] has been proposed in recent years to study molecules and biological tissues. Stokes parameters analysis of SHG has been also recently reported to study the chiral response of metallic nanostructures [28].

Within this context, a two-channel Stokes polarimeter setup has been realized. The experimental apparatus is shown in Fig. 1. The output of a mode-locked femtosecond Ti:sapphire laser system tuned at $\lambda=830 \mathrm{~nm}(76 \mathrm{MHz}$ repetition rate,

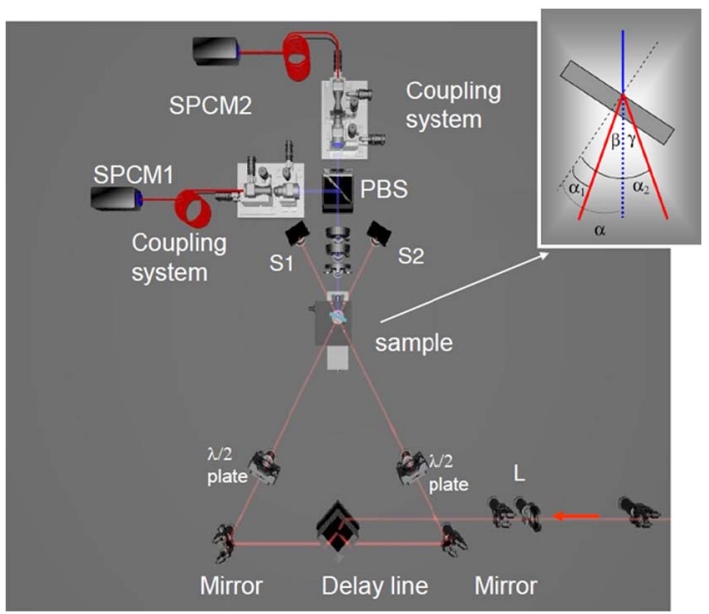

Fig. 1. (Color online) Experimental setup shows the pump light (red light) passing through L (optical lens), then split and delayed by a delay line device. The SHG signal (blue light) passes through a system of filters and quarter-wave plates then is split by a PBS (Thorlabs polarized beam splitter) and sent to SPCM1 (single photon counterPerkinElmer AQ15) and SPCM2 through a fiber-coupling device. Beam stop S1 and S2 suppress the collinear SH signal. The inset shows details of the experimental geometry used for noncollinear SHG. For a given rotation angle $\alpha$, the corresponding incidence angles of the two pump beams result to be $\alpha_{1}=\alpha-\beta$ and $\alpha_{2}=\alpha-\gamma$.
130 fs pulse width, pulse energy of $10 \mathrm{~nJ}$ ) was utilized (red arrow in the Fig. 1). After passing through a collimating lens (L) of $500 \mathrm{~mm}$ focal length, the pump was split into two beams of about the same intensity (Fig. 1: red lines). The sample was placed onto a rotation stage, which allowed for varying the sample rotation angle, $\alpha$, with a resolution of $0.05^{\circ}$. The angle definition is reported in the inset of Fig. 1. The pump beams were sent to intersect in the focus region, on a plane perpendicular to the sample surface, with the angles $\beta=3.5^{\circ}$ and $\gamma=-3.5^{\circ}$, measured with respect to $\alpha=0^{\circ}$. Thus, for a given $\alpha \neq 0^{\circ}$, the corresponding incidence angles of the two pump beams result to be $\alpha_{1}=\alpha-\beta$ and $\alpha_{2}=\alpha-\gamma$, respectively. We set the rotation angle $\alpha$ at $-40^{\circ}$. The SHG direction results along the bisector angle between the two input pump beams, whatever the sample rotation angle. The temporal overlap of the incident pulses was automatically controlled with an external delay line. The polarization of both beams was varied with two identical rotating half-wave plates in the range $-90^{\circ}$ to $+90^{\circ}$ ( $\hat{\mathrm{p}}$-polarized pump beam: $\phi_{1}=0^{\circ}$, and $\hat{\mathrm{s}}$-polarized beam: $\phi_{1}= \pm 90^{\circ}$ ). The average input power of $125 \mathrm{~mW}$ was varied up to $350 \mathrm{~mW}$, arriving at a level of input intensity of $10^{8} \mathrm{~W} / \mathrm{cm}^{2}$.

Two "beam stops" S1 and S2 suppress the collinear SH signals. The coherence length for the SHG process has been estimated of the order of $10 \mu \mathrm{m}$ (for light beams impinging at $-40^{\circ}$ ). A set of optical low-pass filters allows for further suppressing any residual pumping light at $\omega$ frequency. Along the SHG path, the insertion of a quarter wave plate, half-wave plate, and the polarizer beam splitter (PBS) split and control the polarization state of the signals. A suitable rotation of plates allows the projection of the SH signal on the desired polarization state. After splitting, each signal was coupled into two single mode fibers with objective lens (coupling system) and detected by means of a couple of single photon counting detectors (SPCM1 and SPCM2).

Figure 2 shows the polarized SH signals. Each of them was mapped as a function of the linear polarization state of the two input beams $\phi_{1}$ and $\phi_{2}$ at the pump input power of $125 \mathrm{~mW}$. For given values of $\phi_{1}$ and $\phi_{2}$, the maps exhibit the shape and location of the maximum signal values, driven by the symmetry of the material under analysis [13]. The experimental curves were reconstructed using the expression for the effective second-order optical nonlinearity in noncollinear regime and utilizing the nonlinear coefficients listed in the Table 1 . The theoretical signals are just proportional to the experimental one, detected as photon numbers. For this reason, the SHretrieved maps are given in arbitrary units. From the theoretical point of view, the corresponding polarization maps were obtained by changing the nonlinear optical tensor elements to retrieve the location of maxima and minima of the $\mathrm{SH}$ signals and the overall shape of each experimental map. In this procedure, we considered all the detected SH signals, i.e., with the polarizations $\hat{\mathrm{p}}, \hat{\mathrm{s}},+45^{\circ},-45^{\circ}$, RC, and LC polarization. If magnetic dipole and nonlinear magnetic tensor terms are not included into the curve reconstruction, the circular polarization maps exhibit the same symmetric shape. The asymmetries appearing in the SHG maps of $45^{\circ}$ and $-45^{\circ}$ polarization state are mostly influenced by the chiral elements present in the electric dipole tensors. All parameters listed in Table 1 contribute to the shape of the maps. 

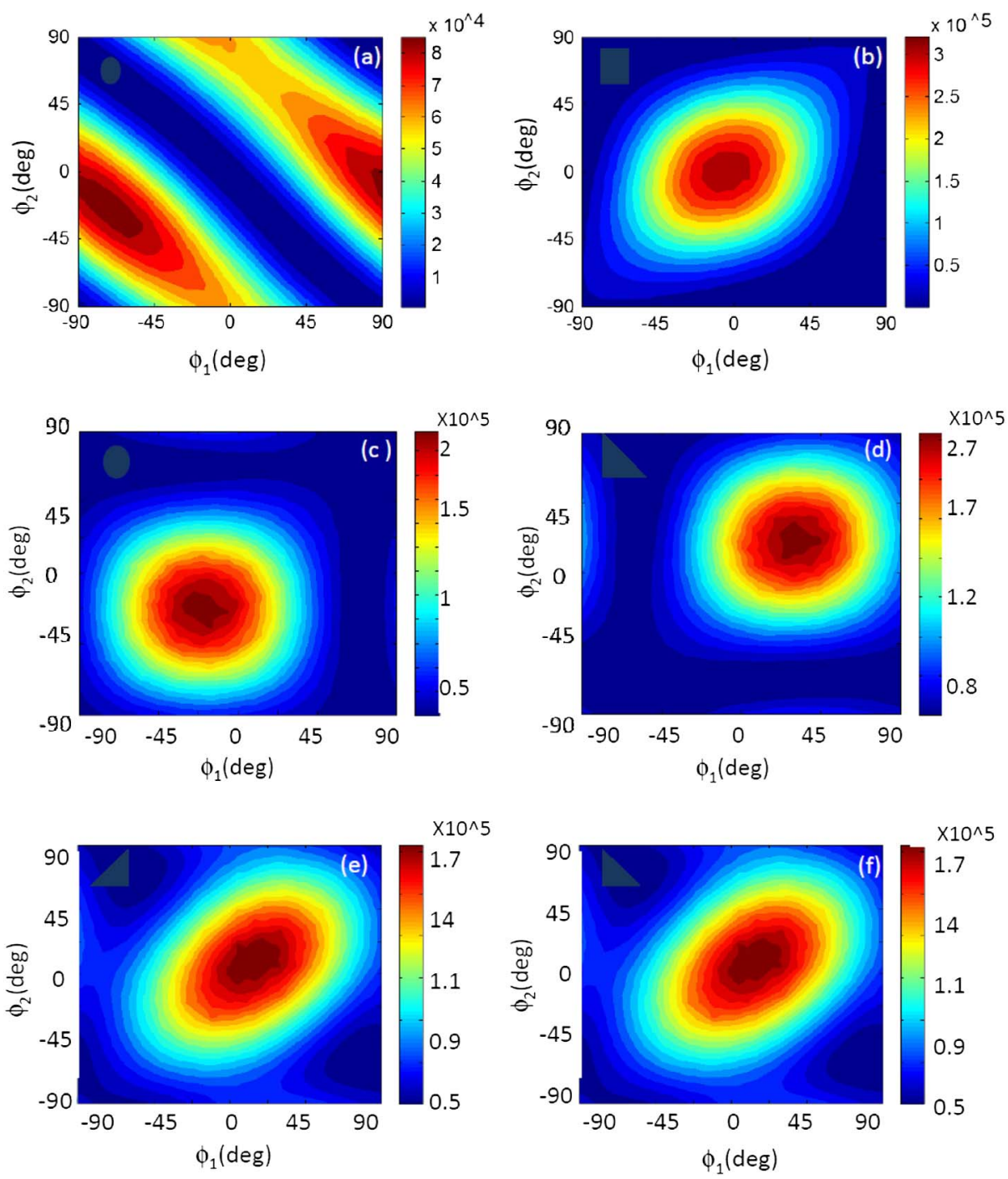

Fig. 2. (Color online) SHG as a function of the polarization state of the first pump beam $\left(\phi_{1}\right)$ and the second pump beam $\left(\phi_{2}\right)$. Sample rotation angle was fixed to $\alpha=-40^{\circ}$. Experimental maps of SH signal intensity (count) are shown for different polarizations: (a) $\hat{\mathrm{s}}$, (b) $\hat{\mathrm{p}}$, (c) $+45^{\circ}$, (d) $-45^{\circ}$, (e) left polarization LC, and (f) right polarization RC.

The full expression of the generated $\mathrm{SH}$ power is a function of sample rotation angle $\alpha$ and is given by $[\underline{13}, \underline{29}]$

$$
\mathrm{I}^{\mathrm{pol}}(\alpha) \simeq F(\alpha) P_{i 1} \cdot P_{i 2} e^{-2\left(\delta_{1}+\delta_{2}\right)}\left(\chi_{\mathrm{eff}}^{\phi_{1} \phi_{2} \rightarrow \mathrm{pol}}(\alpha)\right)^{2},
$$

where superscript (pol) holds for the selected SH signal polarization. $F(\alpha)[\underline{29}]$ is a function containing the fundamental beams' transverse areas onto the sample surface, the Fresnel transmission coefficients for the fundamental fields at the input interface, the Fresnel transmission coefficient for the $\mathrm{SH}$ power at the output interface, and the sample thickness. It also includes a phase factor related to the internal angles of the beams and an additional phase term given by the imaginary part of the refractive index. This part takes into account the effect of absorption on the polarization chart. $P_{i 1}$ and $P_{i 2}$ are the pump beam power. $\left(\chi_{\text {eff }}^{\phi_{1} \phi_{2} \rightarrow \text { pol }}(\alpha)\right)$ is the projection of the tensor on the selected $\mathrm{SH}$ output polarization state at a given $\alpha$ angle:

$\chi_{\mathrm{eff}}^{\phi_{1} \phi_{2} \rightarrow \mathrm{pol}}(\alpha)=\left(\chi_{\mathrm{eff}}^{(e e e)_{\mathrm{pol}}}\right)+\left(\chi_{\mathrm{eff}}^{(e e m 1) \mathrm{pol}}\right)+\left(\chi_{\mathrm{eff}}^{(e e m 2)_{\mathrm{pol}}}\right)+\left(\chi_{\mathrm{eff}}^{(m e e) \mathrm{pol}}\right)$, where all the effective coefficients depend on the input polarization angle, on the nonzero elements of the tensors, according to the above mentioned symmetry class P3, and on the internal propagation angles of the beams inside the slab. As discussed in [13], any change of sign and value of the above mentioned elements modifies the sign and shape of the maps, allowing the possibility to discriminate the weight of the magnetic dipole contribution with respect to the other sources and to evidence the role of the chiral symmetry at the same time.

Each couple of detected polarized SH signals was normalized to the corresponding total intensity according to the following expressions:

$$
\begin{aligned}
& \tilde{S}_{1}\left(2 \omega, \phi_{1}, \phi_{2}\right)=\frac{I^{p}\left(2 \omega, \phi_{1}, \phi_{2}\right)-I^{s}\left(2 \omega, \phi_{1}, \phi_{2}\right)}{I^{p}\left(2 \omega, \phi_{1}, \phi_{2}\right)+I^{s}\left(2 \omega, \phi_{1}, \phi_{2}\right)} \\
& \tilde{S}_{2}\left(2 \omega, \phi_{1}, \phi_{2}\right)=\frac{I^{+45^{\circ}}\left(2 \omega, \phi_{1}, \phi_{2}\right)-I^{-45^{\circ}}\left(2 \omega, \phi_{1}, \phi_{2}\right)}{I^{+45^{\circ}}\left(2 \omega, \phi_{1}, \phi_{2}\right)+I^{-45^{\circ}}\left(2 \omega \phi_{1}, \phi_{2}\right)} \\
& \tilde{S}_{3}\left(2 \omega, \phi_{1}, \phi_{2}\right)=\frac{I^{\mathrm{RC}}\left(2 \omega, \phi_{1}, \phi_{2}\right)-I^{\mathrm{LC}}\left(2 \omega, \phi_{1}, \phi_{2}\right)}{I^{\mathrm{RC}}\left(2 \omega, \phi_{1}, \phi_{2}\right)+I^{\mathrm{LC}}\left(2 \omega \phi_{1}, \phi_{2}\right)},
\end{aligned}
$$



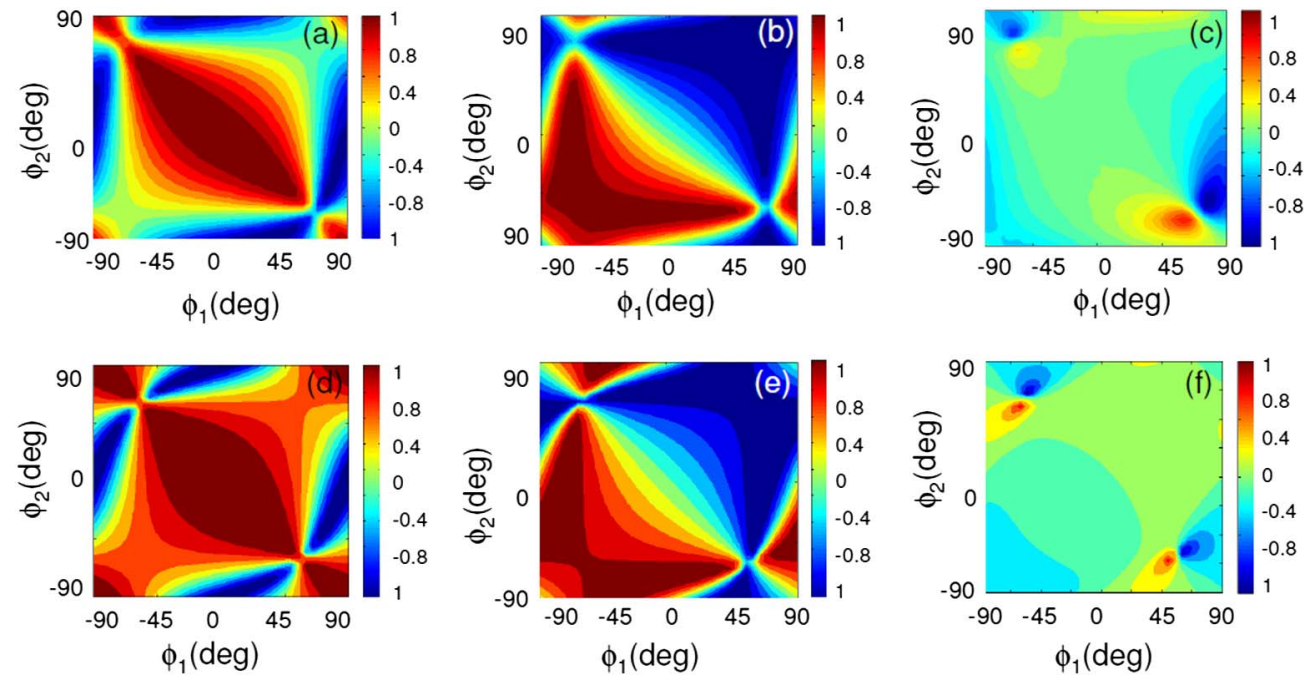

Fig. 3. (Color online) Experimental maps, as a function of the polarization state of the first pump beam $\left(\phi_{1}\right)$ and the second pump beam $\left(\phi_{2}\right)$ of normalized Stokes parameters $\tilde{S}_{1}(\mathrm{a}), \tilde{S}_{2}(\mathrm{~b})$, and $\tilde{S}_{3}$ (c). The input power is $125 \mathrm{~mW}$. Calculated maps $\tilde{S}_{1}$ (d), $\tilde{S}_{2}$ (e), and $\tilde{S}_{3}$ (f) are obtained by using data listed in Table $\underline{1}$.

giving the normalized Stokes parameters, each of them ranging from -1 to 1 , and being $I^{j}\left(j=\hat{\mathrm{s}}, \hat{\mathrm{p}},+45^{\circ},-45^{\circ}, \mathrm{LC}\right.$, $\mathrm{RC})$ the SHG intensity level detected at the $j$-th polarization state.

We verified that, within the experimental errors, the following quantities are equal:

$$
\begin{aligned}
I^{+45^{\circ}} & \left(2 \omega, \phi_{1}, \phi_{2}\right)+I^{-45^{\circ}}\left(2 \omega, \phi_{1}, \phi_{2}\right) \\
& =I^{\mathrm{RC}}\left(2 \omega, \phi_{1}, \phi_{2}\right)+I^{\mathrm{LC}}\left(2 \omega, \phi_{1}, \phi_{2}\right) \\
& =I^{p}\left(2 \omega, \phi_{1}, \phi_{2}\right)+I^{s}\left(2 \omega, \phi_{1}, \phi_{2}\right) .
\end{aligned}
$$

Because the procedure we adopted is based on the polarization change of the two input pumps and on the evaluation of the relative location of maxima and minima signal value on the map, we do not need further calibration procedure.

Figures 3(a)-3(c) show the experimental polarization maps of $\tilde{S}_{1}\left(2 \omega, \phi_{1}, \phi_{2}\right), \widetilde{\tilde{S}_{2}}\left(2 \omega, \phi_{1}, \phi_{2}\right)$, and $\tilde{S}_{3}\left(2 \omega, \phi_{1}, \phi_{2}\right)$ for an input average power of $125 \mathrm{~mW}$. It is worth noting that the presence of nonlinear optical activity gives a nonzero $\tilde{S}_{3}\left(2 \omega, \phi_{1}, \phi_{2}\right)$ map. In fact, when the magnetic dipole interaction is present, the required phase difference between $\mathrm{LC}$ and $\mathrm{RC}$ polarization signals exists also for nonresonant excitation $(\lambda=830 \mathrm{~nm})$. The experimental data have been recovered by analyzing

\begin{tabular}{|c|c|c|c|}
\hline \multicolumn{4}{|c|}{ Magnetic Elements: Nonlinear Coefficients } \\
\hline ұmee & $125 \mathrm{~mW}$ & $250 \mathrm{~mW}$ & $350 \mathrm{~mW}$ \\
\hline$\chi_{x y z}=\chi_{x z y}$ & $0.03+0.06 i$ & $0.03+0.09 i$ & $0.03+0.1 i$ \\
\hline$\chi_{x z x}=\chi_{x z x}$ & $0.06+0.05 i$ & $0.06+0.075 i$ & $0.06+0.1 i$ \\
\hline$\chi_{y y z}=\chi_{y z y}$ & $0.06+0.05 i$ & $0.06+0.075 i$ & $0.06+0.1 i$ \\
\hline$\chi_{y x z}=\chi_{y z x}$ & $-0.03-0.06 i$ & $-0.03-0.09 i$ & $-0.03-0.1 i$ \\
\hline$\chi_{z x x}$ & $0.0009 i$ & $0.0013 i$ & $0.0018 i$ \\
\hline$\chi_{z y y}$ & $0.026 i$ & $0.039 i$ & $0.05 i$ \\
\hline$\chi_{z z z}$ & $0.045 i$ & $0.067 i$ & $0.09 i$ \\
\hline$\chi_{z x y}=\chi_{z y x}$ & 0 & 0 & 0 \\
\hline
\end{tabular}

Table 2. Magnetic Elements $\chi^{m e e}$ at Different Power Levels of 125, 250 and $350 \mathrm{~mW}^{a}$ the $\mathrm{SH}$ signal of $\hat{\mathrm{p}}, \hat{\mathrm{s}},+45^{\circ},-45^{\circ}, \mathrm{RC}$ and $\mathrm{LC}$ polarization. The calculated normalized Stokes parameters [Figs. 3(d)-3(f)] show correctly the location of the maxima and minima values, within the experimental error. These maps range between -1 and +1 , and the recovered data are within an error less than $10 \%$. Nevertheless, the utilized experimental method cannot give the absolute value of the nonlinear coefficients, only relative values. The maps are very sensitive to any change of nonlinear coefficients; for example, relative change of about $10 \%$ of each coefficient modifies the maps. Therefore, the found values of the nonlinear coefficients, listed in Table 1 (and in Table 2), constitute the best fit among the experimental and theoretical maps.

\section{INTENSITY-DEPENDENT SHG}

A very interesting issue of the present work is the possible evidence of the nonlinear magnetic response contribution to the SHG signal, not easily detectable with other methods.

This can be put into evidence by changing the input pump power that induces a detectable change of the nonlinear optical activity, although under nonresonant pump beam excitation.

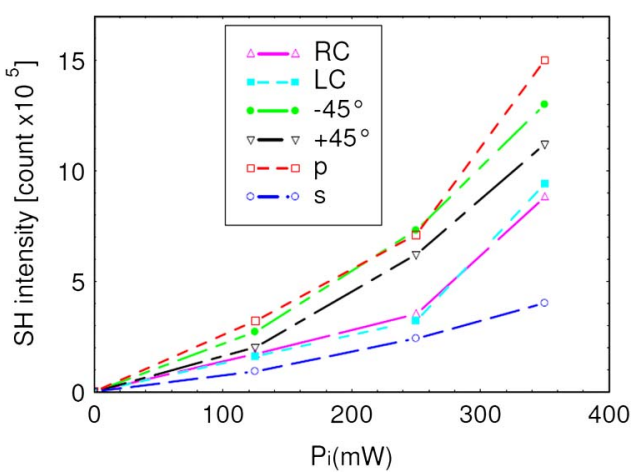

Fig. 4. (Color online) Experimental results showing the maximum SH signal intensity (count) as a function of the input pump power $P_{i}(\mathrm{~mW})$ for each polarization state $\left(\hat{\mathrm{s}}, \hat{\mathrm{p}},+45^{\circ},-45^{\circ}, \mathrm{LC}\right.$, and $\left.\mathrm{RC}\right)$. 

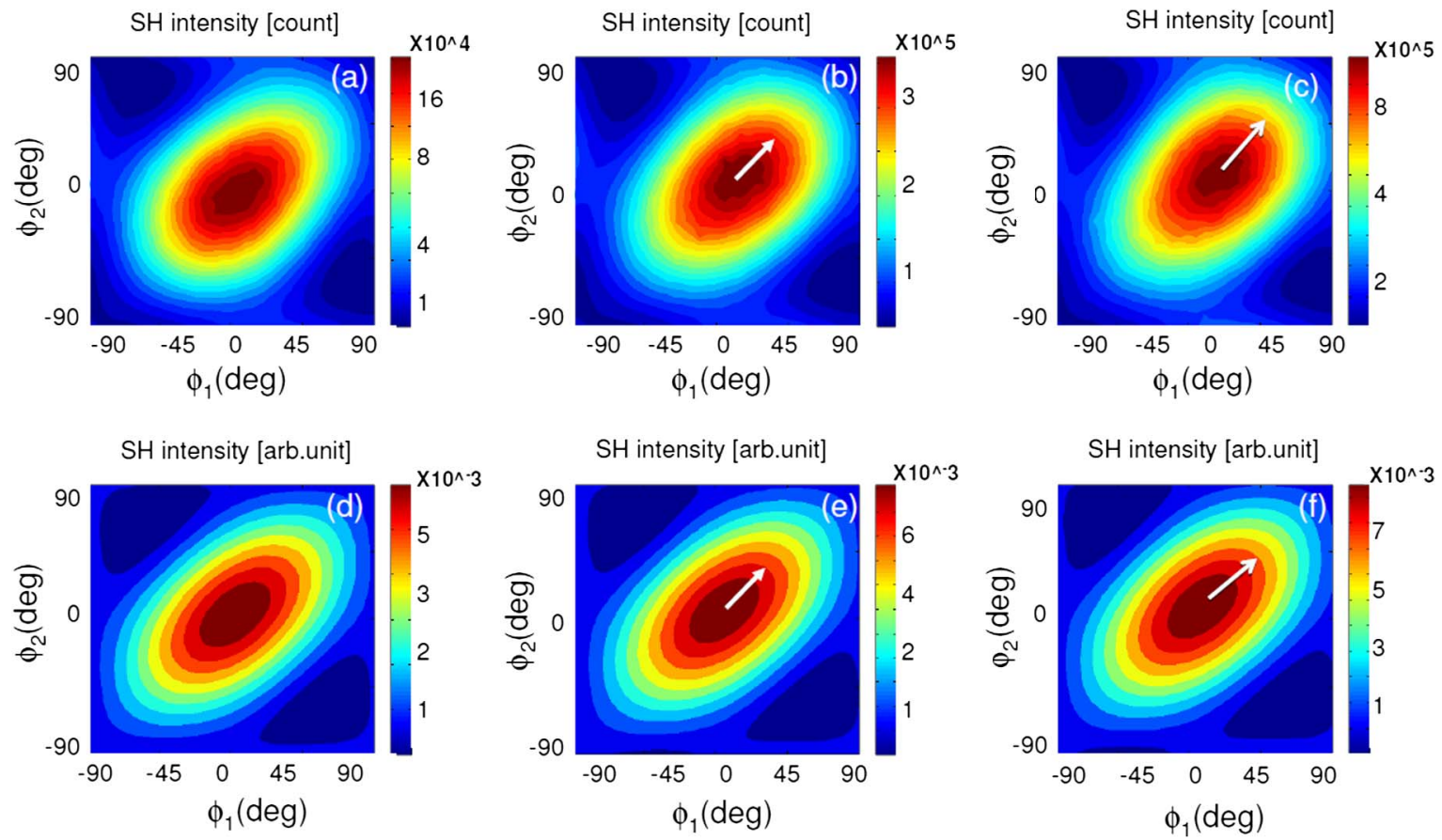

Fig. 5. (Color online) SHG as a function of the polarization state of the first pump beam $\left(\phi_{1}\right)$ and the second pump beam ( $\left.\phi_{2}\right)$ is shown. The experimental LC-polarized SH intensity (count) is presented changing the input pump power: (a) $125 \mathrm{~mW}$, (b) $250 \mathrm{~mW}$, and (c) $350 \mathrm{~mW}$. The theoretically calculated maps (in arbitrary units) are shown in (d) $125 \mathrm{~mW}$, (e) $250 \mathrm{~mW}$, and (f) $350 \mathrm{~mW}$, respectively. The theoretical maps are obtained by using nonlinear coefficients listed in Table 2.

The SHG signal manifests a typical parabolic dependence from the input pump intensity. We have verified this behavior in our system, as shown in Fig. 4, where the maximum SH intensity value is plotted for three levels of the average input power: 125,250 , and $350 \mathrm{~mW}$, and for different output polarization states $\left(\hat{\mathrm{s}}\right.$ and $\hat{\mathrm{p}},+45^{\circ}$ and $-45^{\circ}, \mathrm{LC}$ and $\mathrm{RC}$ polarization).

The polarization maps, expected to be insensitive to the input pump power, are modified as the pump power changes. This is very evident if we follow the circular polarization map. The initial position of the maximum value of the SHG with LC polarization corresponds to an input polarization angle of the pumps of $\phi_{1}=+5^{\circ}$ and $\phi_{2}=+5^{\circ}$, when the input power is $125 \mathrm{~mW}$. As the input power changes, the SHG maximum value position on the map shifts reaching the value of $\phi_{1}=+10^{\circ}$ and $\phi_{2}=+10^{\circ}$, when the input power is $350 \mathrm{~mW}$ [Fig. 5(c)], being as $350 \mathrm{~mW}$ is the highest pump power level in this experiment. Then the trend is a shift of SHG maximum signal location on the map toward positive input polarization angles. The theoretical calculated LC maps, shown in Figs. 5(d)-5(f), are just proportional to the experimental one; for this reason the SH retrieved maps are given in arbitrary units. From the theoretical point of view, those maps were obtained by changing the nonlinear optical tensor elements so as to retrieve the location of maxima and minima SH signals and the overall shape of each experimental map. The nonlinear coefficients found are listed in Table 2 .

The RC polarization SHG shows a similar behavior, however with an opposite trend of the maximum signal location on the map, i.e., the maximum signal value moves toward negative polarization angles. This effect influences the $\tilde{S}_{3}\left(2 \omega, \phi_{1}, \phi_{2}\right)$ map shape, as shown in Fig. $\underline{6}$, for the input power of $125 \mathrm{~mW}$ [Fig. 6(a)], $250 \mathrm{~mW}$ [Fig. 6(b)], and $350 \mathrm{~mW}$ [Fig. 6(c)] respectively. The variation of the input power induces an increase of the difference among $\mathrm{LC}$ and RC SH signals, corresponding to an increase of the "red" and "blue" area on the $\tilde{S}_{3}\left(2 \omega, \phi_{1}, \phi_{2}\right)$ map for a given value of the pump's polarization state. This appears evident when one pump polarization ranges between $45^{\circ}$ and $90^{\circ}$ and the other pump is an ŝ-polarized beam. The same power change also influences $\tilde{S}_{1}\left(2 \omega, \phi_{1}, \phi_{2}\right)$ and $\tilde{S}_{2}\left(2 \omega, \phi_{1}, \phi_{2}\right)$ maps; however, this is less evident with respect to the changes on $\tilde{S}_{3}\left(2 \omega, \phi_{1}, \phi_{2}\right)$ map.

We can summarize the overall power-dependent behavior of the normalized Stokes parameters discussing the ellipticity angle map, defined as $2 \varepsilon=\operatorname{tg}^{-1}\left(\tilde{S}_{3} / \sqrt{\tilde{S}_{1}^{2}+\tilde{S}_{2}^{2}}\right)$. The ellipticity
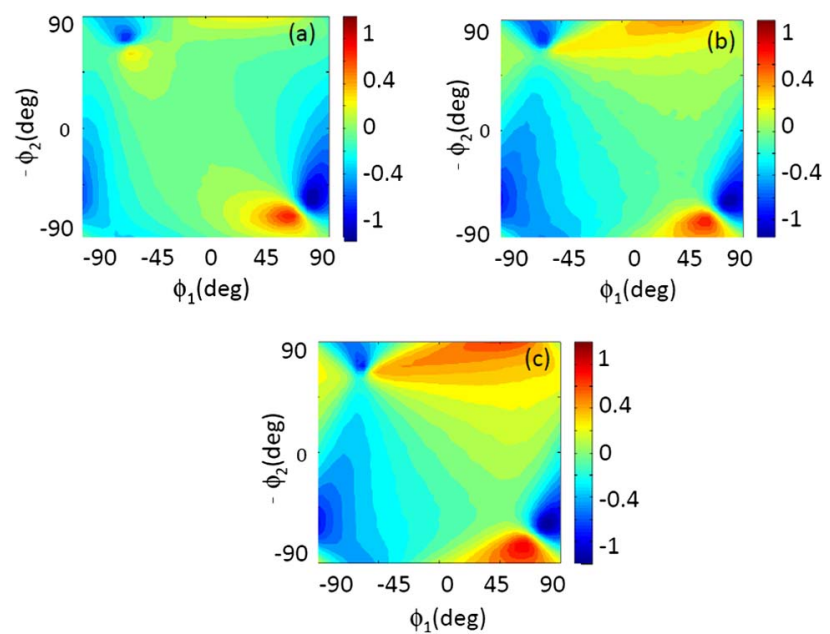

Fig. 6. (Color online) Experimental results showing the $\tilde{S}_{3}$ map as a function of the polarization state of the first pump beam $\left(\phi_{1}\right)$ and the second pump beam $\left(\phi_{2}\right)$, changing the input pump power: (a) $125 \mathrm{~mW}$, (b) $250 \mathrm{~mW}$, and (c) $350 \mathrm{~mW}$. 

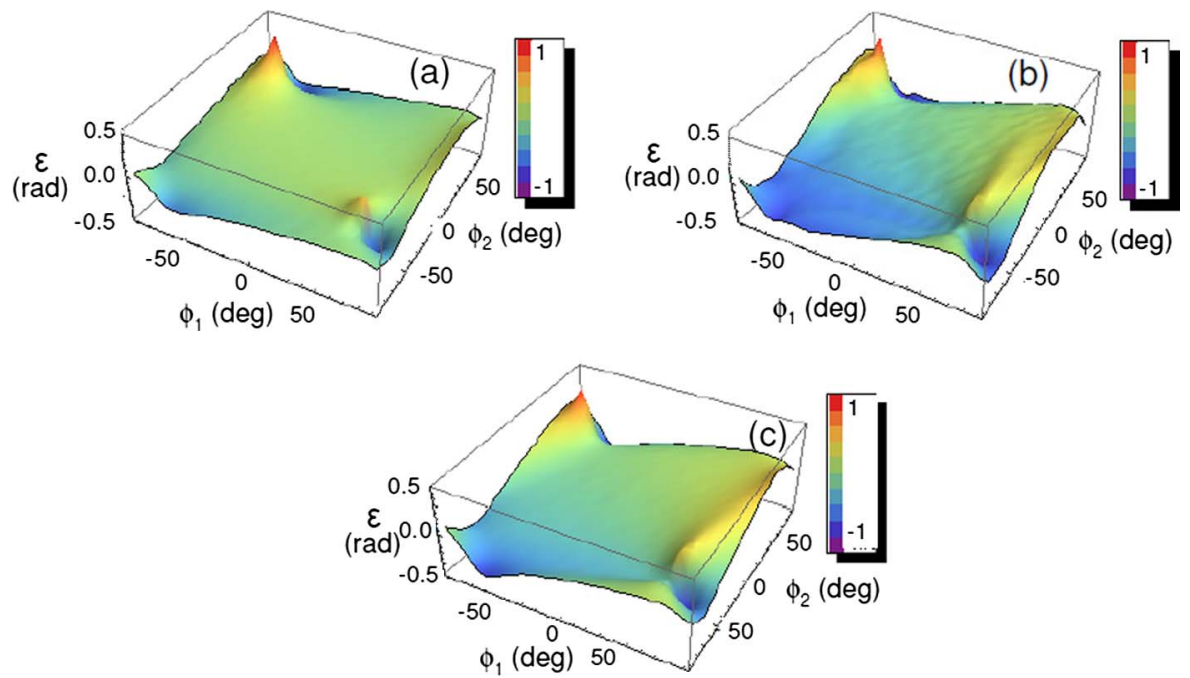

Fig. 7. (Color online) Experimental results showing the ellipticity angle map, ranging from $-\pi / 4$ to $\pi / 4$, as a function of the polarization state of the first pump beam $\left(\phi_{1}\right)$ and the second pump beam $\left(\phi_{2}\right)$, for different input pump power: (a) $125 \mathrm{~mW}$, (b) $250 \mathrm{~mW}$, and (c) $350 \mathrm{~mW}$.

angle $\varepsilon$ puts into evidence the presence of a nonlinear optical activity, typically related to the presence of a magnetic response in the system under study. Figures $7(\mathrm{a})-7(\mathrm{c})$ show the ellipticity map for 125,250 , and $350 \mathrm{~mW}$, respectively. The ellipticity angle ranges from $-\pi / 4$ to $\pi / 4$. Also in this case, for a given value of $\phi_{1}$ and $\phi_{2}$, a power dependence of the ellipticity angle is found. The ellipticity angles are affected by the input power when one polarized beam ranges between $45^{\circ}$ and $90^{\circ}$ and when the other beam is carrying an s-polarized state. This means that the detection of the nonlinear magnetization terms is much more visible for a given set of $\phi_{1}$ and $\phi_{2}$ values.

No power dependent change of $S_{0}$, within the experimental errors, has been observed.

The presented results can be explained according the following considerations.

To recover the experimental results we have included an intensity dependent absorption coefficient at both fundamental and SH frequencies, we have also included the intensity dependent anisotropy [23,24]. However, their changes alone cannot justify the observed results. So we tried to obtain the experimental data assuming an intensity dependence of the nonlinear tensors elements. The experimental results were then recovered by using the values of the nonlinear susceptibility tensors listed in the Tables 1 and 2 . An agreement among the experimental and theoretical maps is found when only the imaginary part of the matrix elements of the magnetic dipole source is found to be dependent from the intensity, indicating an evident nonlinear magnetization contribution to the overall change of the SHG signal.

The origin of the sensitivity of the SHG signal to the input intensity could be attributed to the presence of an electric dipole $\chi^{(4)}$ [23] source; however in our experiment the intensity level of the input beam (of the order of $10^{8} \mathrm{~W} / \mathrm{cm}^{2}$ ) is much lower than what is reported in literature to evidence $\chi^{(4)}$ effects, i.e., from $10^{10} \mathrm{~W} / \mathrm{cm}^{2}$ up to $10^{12} \mathrm{~W} / \mathrm{cm}^{2}$. Also, the nonlinear magnetic sources are affected by the intensity change and not the electric dipole sources.

The observed effect could be ascribed to the thermo-elastic deformation of the BR structure in the cell, averaged over all the sample thickness. Deformation of BR structure as a consequence of piezoelectricity has been reported [24]. The adopted hypothesis is further supported by the "helical path model" [4]: The deformation of the single electron path should influence the nonlinear magnetic response of the chiral material. In fact, according to [4], the ratio among magnetic and electric elements, such as $\chi_{x y z}^{m e e} / \chi_{x y z}^{e e e}$, is proportional to the dimension of the "helical path" ( $\zeta$ and $\rho, \zeta$ being the pitch and $\rho$ the radius of the trajectory, respectively) of the electron moving along the helicoidal trajectory. This gives rise to a sensitivity of the magnetic elements to any variation of the "electron path," i.e., $\zeta=\zeta_{0} \pm \Delta \zeta=\zeta_{0}\left(1 \pm\left(\Delta \zeta / \zeta_{0}\right)\right), \rho=\rho_{0} \pm$ $\Delta \rho=\rho_{0}\left(1 \pm\left(\Delta \rho / \rho_{0}\right)\right)$ assuming the variations $\left(\Delta \zeta / \zeta_{0}\right)$ and $\left(\Delta \rho / \rho_{0}\right)$ proportional to the input power. According to this phenomenological model, a helical path deformation should influence the magnetic dipole elements more than the electric dipole one, with a consequence on the change of the secondorder nonlinear magnetization response, averaged over all the structure.

\section{CONCLUSIONS}

We have reported the SHG from a BR thin film of $4 \mu \mathrm{m}$ thickness. The presented results reveal interesting features related to the nonlinear response of BR excited under a nonresonant regime. We have extended the polarization map method [13], and we have introduced a method based on the polarization map of normalized Stokes parameters of the noncollinear SHG. This method evidences the role of the different nonlinear sources of the nonlinear polarization, as the nonlinear magnetic source. The method reveals the intensity dependence of the only nonlinear magnetic response of BR thin film: a change of input power, although off-resonance, affects the magnetic second-order nonlinear response probably driven by a thermal effects, at a level of lower intensity than the excitation of the electric dipole $\chi^{(4)}$, and making it detectable.

In conclusion the polarization map of Stokes parameters allows a detailed analysis of the SHG signal generated by a chiral dielectric material. The intensity dependent map is a manner to reveal the nonlinear magnetization contribution to the second-order nonlinear polarization in a bulk chiral 
dielectric material, which is not easily separable from the other sources of nonlinear polarization.

\section{REFERENCES}

1. A. Persoon, "Nonlinear optics, chirality, magneto-optics: a serendipitous road," Opt. Mater. Express 1, 5-16 (2011).

2. T. Verbiest, K. Clays, and V. Rodriguez, Second Order Nonlinear Optical Characterization Techniques (CRC, 2009).

3. P. Fischer and F. Hache, "Nonlinear optical spectroscopy of chiral molecules," Chirality 17, 421-437 (2005).

4. J. Maki and A. Persoons, "One electron second order optical activity of a helix," J. Chem. Phys. 104, 9340-9348 (1996).

5. A. V. Rogacheva, V. A. Fedotov, A. S. Schwanecke, and N. I. Zheludev, "Giant gyratory due to electromagnetic field coupling in a bilayered chiral structure," Phys. Rev. Lett. 97, 1-4 (2006).

6. M. Lapine, I. Shadrivov, D. Powell, and Y. Kivshar, "Metamaterials with conformational nonlinearity," Sci. Rep. 138, 1-4 (2011).

7. A. Belardini, M. C. Larciprete, M. Centini, E. Fazio, C. Sibilia, D. Chiappe, C. Martella, A. Toma, M. Giordano, and F. Buatier de Mongeot, "Circular dichroism in the optical second harmonic emission of curved gold metal nanowires," Phys. Rev. Lett. 107, 257401-257404 (2011).

8. A. Belardini, F. Pannone, G. Lehaou, M. C. Larciprete, M. Centini, C. Sibilia, C. Martella, M. Giordano, D. Chiappe, and F. Buatier de Mongeot, "Evidence of anomalous refraction of self-assembled gold nanowires,” Appl. Phys. Lett. 100, 251109 (2012).

9. M. Ren, E. Plum, J. Xu, and N. I. Zhludev, "Giant nonlinear optical activity in a plasmonic metamaterial," Nat. Commun. 3, 833-834 (2012).

10. M. Kauranen, T. Verbiest, J. J. Maki, and A. Persoons, "Second harmonic generation from chiral surfaces," J. Chem. Phys. 101, 8193-8200 (1994).

11. S. Cattaneo and M. Kauranen, "Polarization-based identification of bulk contributions in surface nonlinear optics," Phys. Rev. B 72, 033412 (2005).

12. S. Cattaneo and M. Kauranen, "Polarization techniques for surface nonlinear optics," in Progress in Optics, E. Wolf, ed. (Elsevier, 2008), pp. 69-101.

13. F. A. Bovino, M. C. Larciprete, C. Sibilia, G. Varo, and C. Gergely, "Evidence of multipolar response of bacteriorhodopsin by noncollinear second harmonic generation," Opt. Express 20, 14621-14631 (2012).

14. T. Verbiest, S. Sioncke, and A. Persoon, "Magnetic-dipole nonlinearities in chiral materials," J. Photochem. Photobiol. A 145, 113-115 (2001).

15. C. Gergely, L. Zimányi, and G. Váró, "Bacteriorhodopsin intermediate spectra determined over a wide $\mathrm{pH}$ range," J. Phys. Chem. B 101, 9390-9395 (1997).
16. R. Wampler, M. Zhou, D. H. Thomson, and G. J. Simpson, "Mechanism of the chiral SHG activity of bacteriorhodopsin films," J. Am. Chem. Soc. 128, 10994-10995 (2006).

17. F. A. Bovino, M. C. Larciprete, M. Giardina, and C. Sibilia, "Method and system for determining second-order nonlinear optical coefficients," patent EP2414893, USP 20120158366 (August 2, 2010).

18. M. C. Larciprete, F. A. Bovino, M. Giardina, A. Belardini, M. Centini, C. Sibilia, M. Bertolotti, A. Passaseo, and V. Tasco, "Mapping the nonlinear optical susceptibility by noncollinear second-harmonic generation," Opt. Lett. 34, 2189-2191 (2009).

19. F. A. Bovino, M. C. Larciprete, A. Belardini, and C. Sibilia, "Evaluation of the optical axis tilt of zinc oxide films via noncollinear second harmonic generation," Appl. Phys. Lett. 94, 251109 (2009).

20. D. Oesterhelt, J. Tittor, and E. Bamberg, "A unifying concept for ion translocation by retinal proteins," J. Bioenerg. Biomembr. 24, 181-191 (1992).

21. W. Stoeckenius, R. H. Lozier, and R. A. Bogomolni, "Bacteriorhodopsin and the purple membrane of alobacteria," Biochim. Biophys. Acta 505, 215-278 (1979).

22. M. C. Larciprete, A. Belardini, C. Sibilia, M. B. Saab, G. Varo, and C. Gergely, "Optical chirality of bacteriorhodopsin films via second harmonic maker's fringes measurements," Appl. Phys. Lett. 96, 221108 (2010).

23. Q. Wang Song, C. Zhang, R. Gross, and R. Birge, "Optical limiting by chemically enhanced bacteriorhodopsin films," Opt. Lett. 18, 775-777 (1993).

24. O. Bouevitch and A. Lewis, "Probing bacteriorhodopsin photochemistry with nonlinear optics: comparing the second harmonic generation of $\mathrm{bR}$ and the photochemically induced intermediate K," Opt. Commun. 116, 170-174 (1995).

25. Z. Zhang, Q. W. Song, C. Y. Ku, R. B. Gross, and R. R. Birge, "Determination of the refractive index of a bacteriorhodopsin film," Opt. Lett. 19, 1409-1411 (1994).

26. S. Brasselet, "Polarization-resolved nonlinear microscopy: application to structural molecular and biological imaging," Adv. Opt. Photonics 3, 205-271 (2011).

27. N. J. Begue, M. Everly, V. J. Hall, L. Haupert, and G. J. Simpson, "Nonlinear optical stokes ellipsometry 2. Experimental demonstration," J. Phys. Chem. C 113, 10166-10175 (2009).

28. E. A. Mamonov, T. V. Murzina, I. A. Kolmychek, A. I. Maydykovsky, V. K. Valev, A. V. Silhanek, T. Verbiest, V. V. Moshchalkov, and O. A Aktsipetrov, "Chirality in nonlinear optical response of planar G-shaped nanostructures,” Opt. Express 20, 8518-8523 (2012).

29. M. C. Larciprete, F. A. Bovino, A. Belardini, C. Sibilia, and M. Bertolotti, "Bound and free waves in non-collinear second harmonic generation,” Opt. Express 17, 17000-17009 (2009). 